\section{A challenging case of neuropsychiatric systematic lupus erythematosus with recurrent antiphospho- lipid-related stroke: A case report and literature review}

\author{
Yu-Cheng Shih, ${ }^{1}$ Yang-Hao Ou, ${ }^{1}$ \\ Shu-Wei Chang, ${ }^{2}$ Chih-Ming Lin ${ }^{1}$
}

1Department of Neurology, Changhua Christian Hospital; 2Department of Medicinal Botanicals and Health Applications, Dayeh University, Taiwan

\section{Abstract}

Neuropsychiatic systematic lupus erythematosus (NPSLE) is a form of SLE involves the inflammation and/or thrombotic event in the nervous system. Patients with NPSLE are likely to have a positive antiphospholipid antibody (aPL), therefore are at higher risk of recurrent ischemic stroke. The management of NPSLE with aPLrelated stroke is rather different from the traditional ischemic stroke. One must treat it with anticoagulation and immunosuppressive therapy. The present case is a 47-yearold Taiwanese female with NPSLE and positive aPL, presented with a recurrent MCA ischemic stroke. Initial laboratory results showed significantly elevated levels of anti-ANA, anti-dsDNA, anti-cardiolipin, and decreased complement levels. Due to multiple contraindications for tPA, she was treated with antiplatelet, anticoagulation, steroid pulse therapy, and plasmapheresis during the hospitalization. Despite treatments, her stroke progressed to multi-focal lesions, involving the ACA, MCA, and basal ganglion. On follow up of her brain CT scan showed tissue edema and suspicious for subfalcine herniation. Responding to this clinical deterioration, we stopped warfarin and started mannitol. Eventually, her condition improved and was transferred to the rehabilitation program. Currently, there is no unified guideline regarding the secondary prevention of ischemic stroke in NPSLE with aPL patients. Additionally, previously reported use of steroid pulse therapy and plasmapheresis can potentially harm the patient. Clinicians must be cautious when treating such patient.

\section{Introduction}

Neuropsychiatric systemic lupus ery- thematosus (NPSLE) is a clinical manifestation of SLE that involves the central and/or peripheral nervous system as the result of autoimmune or inflammatory nature of the disease. ${ }^{1}$ It is not an uncommon clinical manifestation in patients with SLE, and there is a plethora of presentation, ranging from headache, mood disorders, to cerebrovascular diseases. ${ }^{2}$ NPSLE can further categorized into medium-to-large vessel pathologies such as atherosclerosis or thromboembolism, ${ }^{3}$ and inflammation or immune-deposition of the small vessels. ${ }^{4}$

SLE alone increases the risk for cerebrovascular disease by eight-fold when compared with the general population, 5 and the presence of antiphospholipid antibody (aPL) amplifies the risk further. It has been estimated that thirty to forty percent of SLE patients have a positive aPL profile, ${ }^{6}$ which contributes to a higher risk of ischemic stroke $^{7}$ and a relevant risk of recurrent thrombotic events. ${ }^{8-10}$ NPSLE patients with positive aPL pose a challenge to clinical management; studies have shown that using antiplatelet and/or anticoagulant as secondary prevention of stroke have a relatively high failure risk. ${ }^{11}$ Bazzan et al. reported that as high as 9.6\%/patient/year have recurrent thrombosis despite medical prevention. ${ }^{11}$ Here we present a 47-year-old Taiwanese female NPSLE patient with positive aPL presented with a recurrent largevessel ischemic stroke despite the secondary prevention with oral anticoagulation. Furthermore, a rapid progression into multilarge-vessel stroke with the initial treatment of steroid pulse therapy. Followed by a literature review of the current guidelines in managing such patient.

\section{Case Report}

A 47-year-old Taiwanese female patient with a past medical history of SLE, hypertension, and type 2 diabetes. For the past ten years, her SLE symptoms have been adequately controlled with methylprednisolone $8 \mathrm{mg}$ daily and azathioprine $50 \mathrm{mg}$ everyother-day. However, one month ago, she was admitted to one of our satellite hospitals with a diagnosis of acute ischemic stroke of the right middle cerebral artery (MCA) territory and suffered from left hemiparesis leg. At the same time, she was diagnosed with positive antiphospholipid antibody and was treated with aspirin 100 $\mathrm{mg}$ and warfarin $5 \mathrm{mg}$ for secondary prevention of cerebrovascular diseases. The international normalized ratio (INR) on discharged was 1.94 .

At present, she presented to Changhua Christian Hospital with new-onset
Correspondence: Chih-Ming Lin, $\mathrm{MD}, \mathrm{PhD}$, Department of Neurology, Changhua Christian Hospital, No. 135, Nanxiao Street, Changhua City, Changhua County 500, Taiwan (R.O.C.)

Tel.: 886-4-7238595 ext. 4227

E-mail: 166110@cch.org.tw

Key words: acute ischemic stroke, neuropsychiatric systemic lupus erythematosus, Antiphospholipid syndrome, pulse therapy, plasmapheresis.

Contributions:YCS and YHO contributed equally to this work.

Conflict of interest: the authors declare no potential conflict of interest.

Funding: none.

Received for publication: 21 May 2019

Accepted for publication: 10 June 2019.

This work is licensed under a Creative Commons Attribution NonCommercial 4.0 License (CC BY-NC 4.0).

(C) Copyright: the Author(s), 2019

Licensee PAGEPress, Italy

Neurology International 2019; 11:8182

doi:10.4081/ni.2019.8182

headache, skin rash over the right thigh, and right leg weakness. Her initial physical exam was significant for decreased muscle strength of the right leg (MRC: upper limb $5 / 5$, lower limb 2/5), left hemiparesis (MRC: $3 / 5$ on upper and lower limbs) as the sequelae from the previous stroke. There were no abnormal findings in her cognitive, cerebellum, or cranial nerve functions. Initial laboratory results were significant for elevated erythrocyte sedimentation rate (ESR, $87 \mathrm{~mm} / \mathrm{hr}$ ), and a subtherapeutic INR of 1.39 .

DWI sequence of brain MRI on admission revealed multifocal acute cortical infarctions involving the right frontal, temporal, and parietal lobes, along the MCA territories (Figure 1). However, the MRI findings were inconsistent with her current clinical manifestation, and therefore, we suspected autoimmune processes related to SLE. Checking her immunological profiles revealed elevated levels of anti-nuclear antibody, anti-dsDNA antibody, anti- $\beta 2$ glycoprotein I IgM and IgG, anti-SSA, and anti-Cardiolipin IgG (Table 1). Complement levels were decreased and negative reactivity for anti-Sm, anti-SSB, and anti-ribosomal $\mathrm{P}$ antibody. Coagulopathy panel results were not significant. Subsequently, we started her on a 
pulse therapy with methylprednisolone $750 \mathrm{mg}$, plasmapheresis for NPSLE vasculitis-related stroke and continued with anticoagulation therapy. Despite treatment, we noticed worsening of her left hemiparesis (MRC: 0-1), diminished social interaction and flat affect. A repeat MRI of the brain was performed on day 5 of hospitalization and showed new lesion on the right basal ganglion and right frontal lobe as the result of acute hemorrhagic transformation involving the anterior cerebral artery (ACA) and MCA (Figure 2). At this point, we discontinued warfarin but kept aspirin. A follow up of the brain CT scan (Figure 3) on day 10 showed signs suspicious for subfalcine herniation, extensive multifocal acute infarction of the right basal ganglion, right $\mathrm{ACA}$, right $\mathrm{MCA}$, and tissue edema. At this time, mannitol $100 \mathrm{mg}$ was added to prevent further progression of the edema. With careful monitoring and follow up CT showed reduced brain edema, the patient was transferred to rehabilitation on day 17 with hemiplegia of the left upper and lower limbs (MRC:1).

\section{Discussion}

NPSLE presents with a higher risk of cerebrovascular injury for multiple mechanisms associated with the presence of aPL; the binding of aPL to endothelial cells contribute to the activation of endothelial cells and other inflammatory cells such as neutrophils and monocytes and therefore increase the production of inflammatory cytokines. 12 aPL favors clot formation of the platelet by increasing the expression of glycoprotein IIb/IIIa. ${ }^{12}$ As a result, patients with NPSLE have a higher prevalence of vasculopathy and accelerated atherosclerosis. ${ }^{1}$ Our patient presents with two clinical challenges related to the underline pathogenesis of NPSLE, and to-date there is no clear guideline on managing of both scenarios.

First, the treatment for the inflammatory process of NPSLE with the use of steroid pulse therapy ${ }^{13,14}$ and plasmapheresis ${ }^{12,15}$ have been reported successful in ischemic stroke patients. Despite the rapid treatment with anticoagulation, steroid pulse therapy, and plasmapheresis, our patient developed yet another ischemic stroke involving the ACA and MCA with subsequent hemorrhagic transformation and brain edema. Currently, there is no evidence indicating the use of steroid pulse therapy or plasmapheresis led to the progression of the NPSLE ischemic stroke. However, we suspect the possibility that plasmapheresis alters the dynamics of cerebral blood flow and somehow led to extensive ischemic stroke.

Secondly, the use of anticoagulant for secondary prevention of cerebrovascular injury in patients with aPL has no consensus among experts. Some studies have suggested that aspirin alone is sufficient, 16 while others advocate the use of warfarin with or without aspirin.17 According to the
Galveston guidelines, the current recommendation for target INR should be at a higher level of 3.0 to 4.0 for warfarin alone, or INR of 2.0 to 3.0 when the combination of aspirin and warfarin are used. 18 However, a systematic review has reported a high recurrent arterial event in patients with target INR of 2.0 to 3.0, and therefore advocate for aggressive anticoagulation

Table 1. Immunological profiles.

\begin{tabular}{lcc} 
Measurements & Results & Reference Range \\
ANA: Homogeneous pattern & $1: 640$ & $<1: 160$ \\
ANA: Anti-cytoplasmic staining & $1: 40$ & $<1: 160$ \\
\hline Anti-dsDNA antibody & 278.1 & $<92.6 \mathrm{WHO} \mathrm{units} / \mathrm{mL}$ \\
Anti-2 glycoprotein I IgM & 0.3 & $<20.0 \mathrm{u} / \mathrm{mL}$ \\
\hline Anti- 2 glycoprotein I IgG & $>160$ & $<20.0 \mathrm{u} / \mathrm{mL}$ \\
Anti-SSA & $>8.0$ & $<1.0$ \\
\hline Anti-SSB & 0.4 & $<1.0$ \\
Anti-Sm & 0.7 & $<1.0$ \\
\hline Anti-RNP & 0.4 & $<1.0$ \\
Anti-Cardiolipin IgG & $>160.0$ & $<20 \mathrm{GPL}-\mathrm{U} / \mathrm{mL}$ \\
\hline Anti-Cardiolipin IgM & 0.2 & $<20 \mathrm{MPL}-\mathrm{U} / \mathrm{mL}$ \\
Rapid plasma reagin (RPR) & $\mathrm{NR}$ & $\mathrm{NR}$ \\
\hline T. pallidum Ab confirmation & Negative & Negative \\
C3C & 89.2 & $90-180 \mathrm{mg} / \mathrm{dL}$ \\
\hline C4 & $<6.4$ & $10-40 \mathrm{mg} / \mathrm{dL}$ \\
Antithrombin function & 120.4 & $87-130 \%$ \\
\hline Factor V activity & 115.0 & $78-151 \%$ \\
Protein S function & 61.9 & $63.5-149 \%$ \\
\hline Protein C function & 99.4 & $79-152 \%$ \\
\hline
\end{tabular}
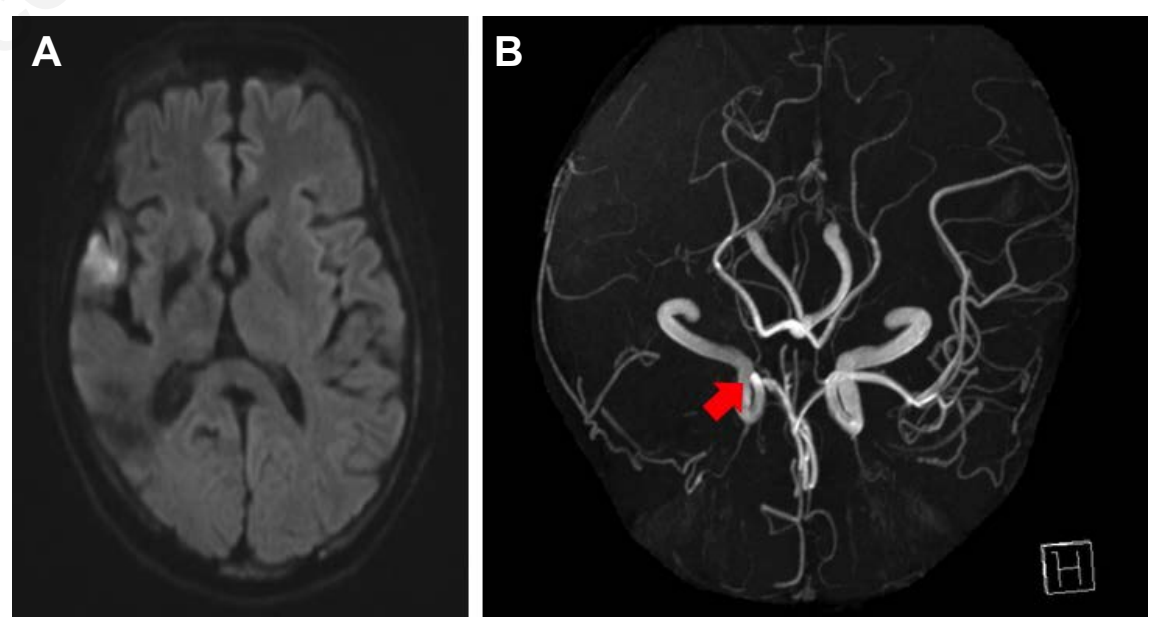

Figure 1. A) Multifocal acute cortical infarctions involving the right MCA frontotemporoparietal lobes cortical territories; no focal acute hemorrhagic transformation complication nor acute brain herniation occurs. Multifocal brain tissues loss in the right basal ganglion, right frontal corona radiata white matters and right frontotemporal lobes MCA cortical territories as the result of previous infarction. The right lateral ventricle shows focal passive atrophic dilatation and early right corticospinal tract Wallerian degenerative change. B) Total occlusion of the right MCA from its proximal M1 segment and no normal antegrade flow over its right M2-4 segments. 
strategy. ${ }^{19}$ Additionally, patient with positive aPL is less likely to have a recurrent arterial event if INR $>3.0$ is achieved. ${ }^{20}$

It is important to distinguish the underlying process of the disease, whether it be inflammatory or thrombotic, as a guide for the intervention; however, it is often diffi- cult, and two processes can co-exist. The present case illustrates a dilemma in the clinical decision; The patient had difficulty in achieving the therapeutic INR, regardless of the target ranges. Furthermore, her stroke has progressed to hemorrhagic transformation after plasmapheresis. We consulted
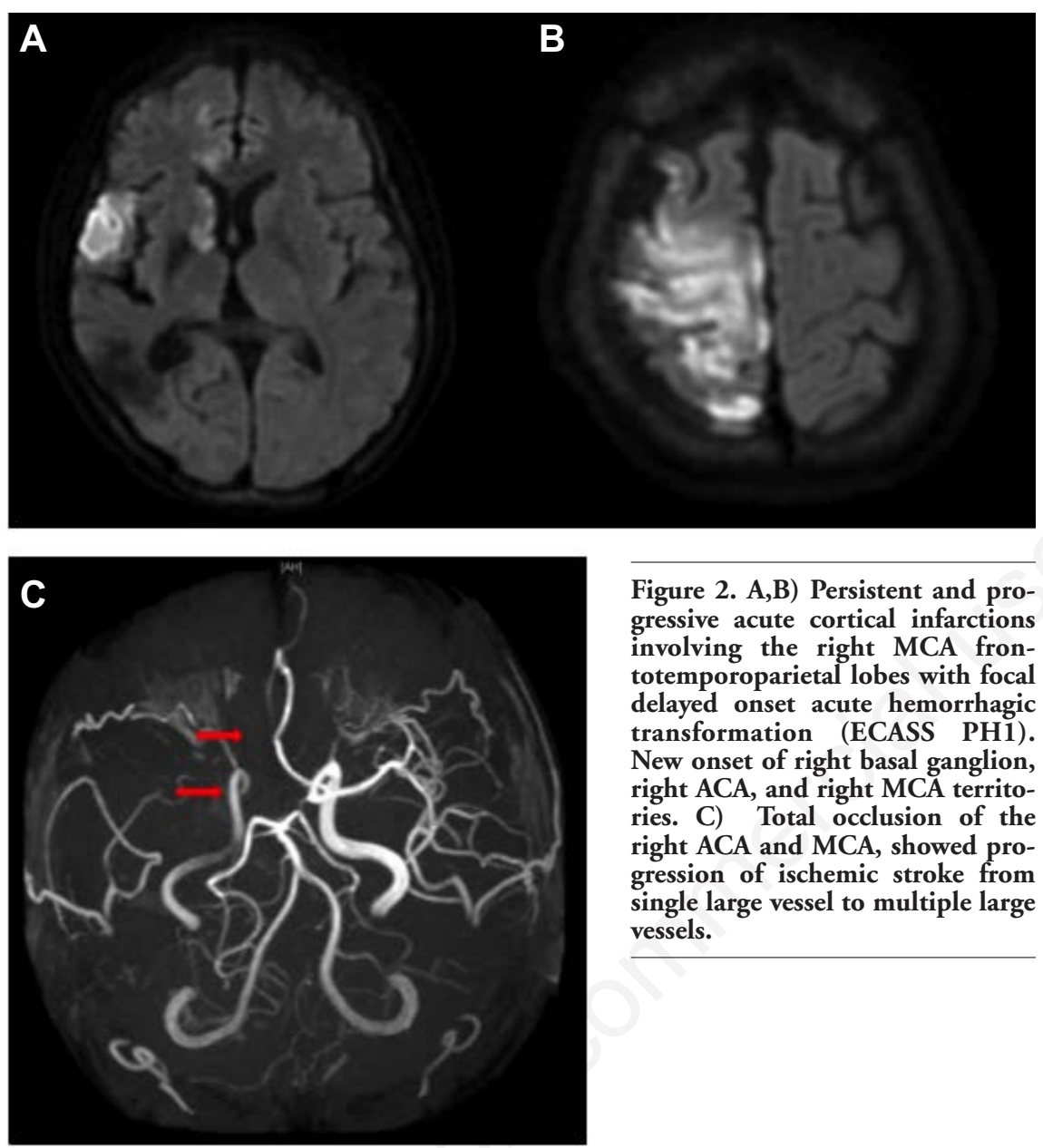

Figure 2. A,B) Persistent and progressive acute cortical infarctions involving the right MCA frontotemporoparietal lobes with focal delayed onset acute hemorrhagic transformation (ECASS PH1). New onset of right basal ganglion, right ACA, and right MCA territories. C) Total occlusion of the right ACA and MCA, showed progression of ischemic stroke from single large vessel to multiple large vessels.
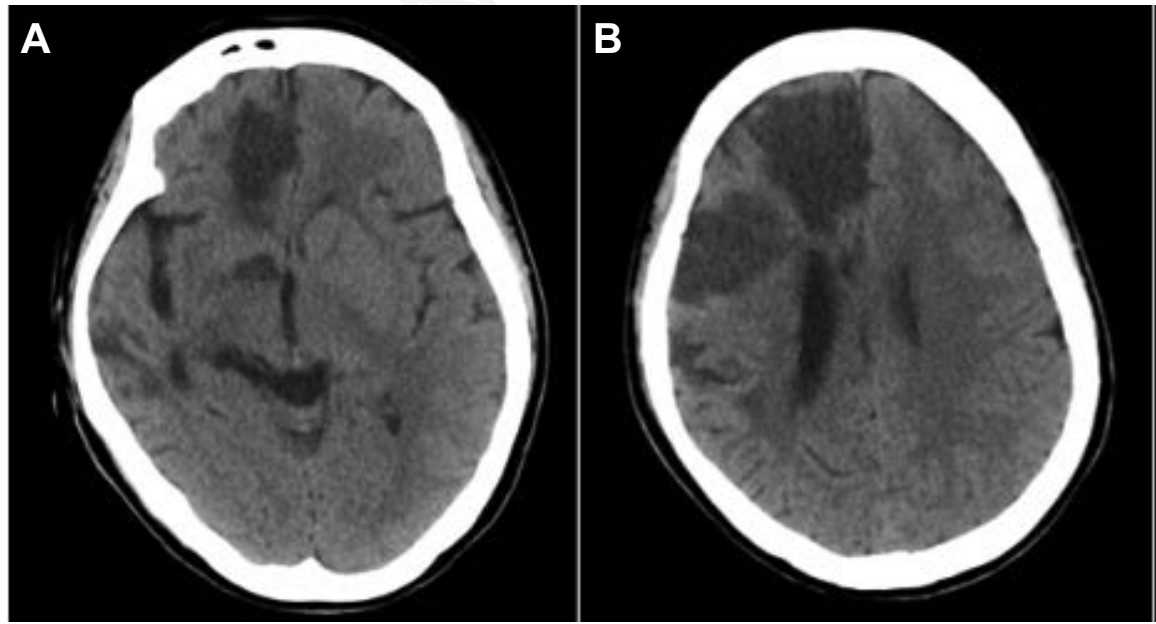

experts in neurology and rheumatology on the strategy for anticoagulation. However, there was no consensus whether we should hold anticoagulation or proceed until we have reached the target INR. After all, with the fear for worsening of cerebral edema, we decided to hold warfarin but kept aspirin, statin, and started patient on mannitol.

\section{Conclusions}

NPSLE patients present with a higher risk of initial and recurrent arterial events. While the use of anticoagulation for secondary prevention is the mainstay of treatment, however, there is no clear guideline regarding the method and INR goal of anticoagulation. Additionally, it is prudent to treat both inflammatory and thrombotic process during an acute event; however, we must monitor the patient carefully when initiating plasmapheresis. Future researches should be aimed at unifying the guideline for secondary prevention of arterial thrombotic event and elucidate whether plasmapheresis is beneficial in such patients.

\section{References}

1. Bertsias GK, Boumpas DT. Pathogenesis, Diagnosis and Management of Neuropsychiatric SLE Manifestations. Nat Rev Rheumatol 2010;6:358-67.

2. The American College of Rheumatology Nomenclature and Case Definitions for Neuropsychiatric Lupus Syndromes. Arthritis Rheum

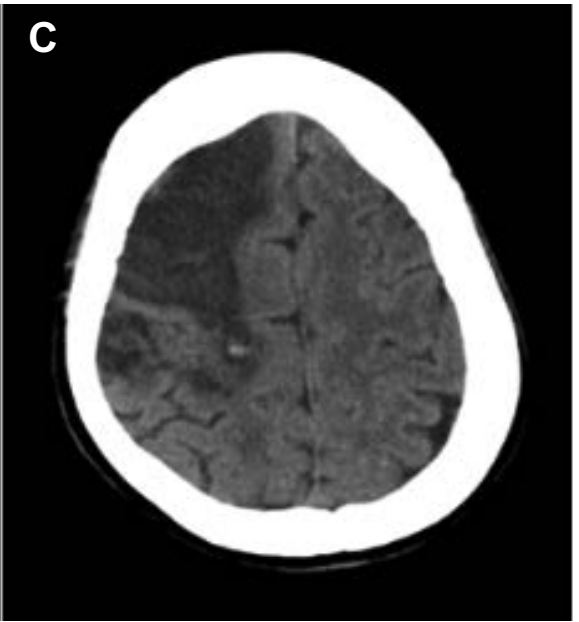

Figure 3. CT scan of the head showed extensive multifocal right basal ganglion, right ACA, and right MCA infarction with brain edema and suspected for subfalcine herniation. 
1999;42:599-608.

3. Kitagawa Y, Gotoh F, Koto A, Okayasu H. Stroke in Systemic Lupus Erythematosus. Stroke 1990;21:1533-9.

4. Hess DC. Cerebral Lupus Vasculopathy. Mechanisms and Clinical Relevance. Ann NY Acad Sci 1997;823:154.

5. Esdaile JM, Abrahamowicz M, Grodzicky T, et al. Traditional Framingham Risk Factors Fail to Fully Account for Accelerated Atherosclerosis in Systemic Lupus Erythematosus. Arthritis Rheum 2001;44:2331-7.

6. Ünlü O, Zuily S, Erkan D. The clinical significance of antiphospholipid antibodies in systemic lupus erythematosus. Eur J Rheumatol 2016;3:75-84.

7. de Amorim LCD, Maia FM, Rodrigues CEM. Stroke in Systemic Lupus Erythematosus and Antiphospholipid Syndrome: Risk Factors, Clinical Manifestations, Neuroimaging, and Treatment. Lupus 2017;26:529.

8. Sciascia S, Sanna G, Khamashta MA, et al. The Estimated Frequency of Antiphospholipid Antibodies in Young Adults with Cerebrovascular Events: A Systematic Review. Ann Rheum Dis 2015;74:2028-33.

9. Gerosa M, Meroni PL, Erkan D. Recognition and Management of Antiphospholipid Syndrome. Curr Opin
Rheumatol 2016;28:51-9.

10. Erkan D, Harrison MJ, Levy R, et al. Aspirin for Primary Thrombosis Prevention in the Antiphospholipid Syndrome: A Randomized, DoubleBlind, Placebo-Controlled Trial in Asymptomatic Antiphospholipid Antibody-Positive Individuals. Arthritis Rheum 2007;56:2382.

11. Bazzan M, Vaccarino A, Stella S, et al. Thrombotic Recurrences and Bleeding Events in APS Vascular Patients: A Review from the Literature and a Comparison with the APS Piedmont Cohort. Autoimmun Rev 2013;12:82631.

12. David G, Erkan D. Diagnosis and Management of the Antiphospholipid Syndrome. New Eng J Med 2018;378:2010.

13. Ishitsuka K, Ago T, Fukuda K et al. A case of SLE presenting stroke-like symptoms. Intern Med 2011;50:359-62

14. Sideras PA, Jacob J, Mann A, et al. Middle Cerebral Artery Involvement in Systemic Lupus Erythematosus Presenting as a Stroke - an Unusual Initial Presentation of the Disease. J Clin Neurosci 2012;19:1463-4.

15. Seifert CL, Kowarik MC, Thürmel K, et al. Rapidly Progressive Intracranial Artery Stenosis in Primary Antiphospholipid Syndrome. Ann Clin
Translat Neurol 2015;2:780-2.

16. Levine SR, Brey RL, Tilley BC, et al. Antiphospholipid Antibodies and Subsequent Thrombo-Occlusive Events in Patients with Ischemic Stroke. JAMA 2004;291:576.

17. Keeling D, Mackie I, Moore GW, et al. Guidelines on the Investigation and Management of Antiphospholipid Syndrome. Br J Haematol 2012;157:4758.

18. Ruiz-Irastorza G, Cuadrado MJ, RuizArruza I, et al. Evidence-Based Recommendations for the Prevention and Long-Term Management of Thrombosis in Antiphospholipid Antibody-Positive Patients: Report of a Task Force at the 13th International Congress on Antiphospholipid Antibodies. Lupus 2011;20:206-18.

19. Ruiz-Irastorza G, Hunt BJ, Khamashta MA. A Systematic Review of Secondary Thromboprophylaxis in Patients with Antiphospholipid Antibodies. Arthritis Rheum 2007; 15:1487-95.

20. Tan BE, Thong BY, Shivananda S, et al. Clinical Manifestations and Outcomes of Antithrombotic Treatment of the Tan Tock Seng Hospital Singapore Antiphospholipid Syndrome Cohort. Lupus 2009;18:752-8. 